Recherches en didactique des langues et des cultures

Les cahiers de l'Acedle

$13-2 \mid 2016$

Interactions langagières et didactique des langues

\title{
L'alternance codique entre L2 et L3
}

Quelques remarques sur le contrôle des systèmes plurilingues

Teresa Maria Wlosowicz

\section{OpenEdition}

1 Journals

Édition électronique

URL : http://journals.openedition.org/rdlc/892

DOI : $10.4000 /$ rdlc.892

ISSN : $1958-5772$

Éditeur

ACEDLE

Référence électronique

Teresa Maria Wlosowicz, "L'alternance codique entre L2 et L3 », Recherches en didactique des langues et des cultures [En ligne], 13-2 | 2016, mis en ligne le 24 octobre 2016, consulté le 19 avril 2019. URL: http://journals.openedition.org/rdlc/892 ; DOI : 10.4000/rdlc.892

Ce document a été généré automatiquement le 19 avril 2019

\section{(c) $($ ) $(9)$}

Recherches en didactique des langues et des cultures is licensed under a Creative Commons AttributionNonCommercial-NoDerivatives 4.0 International License 


\title{
L'alternance codique entre L2 et L3
}

Quelques remarques sur le contrôle des systèmes plurilingues

\author{
Teresa Maria Wlosowicz
}

\section{Introduction}

1 Le but de l'étude est une investigation de l'alternance codique entre L2 et L3 dans plusieurs combinaisons de langues. Or, à part les interactions interlinguales de plusieurs types, c'est-à-dire, le transfert, les interférences, les emprunts, etc. (cf. Herdina \& Jessner, $2002: 29$, et la notion de "cross-linguistic interaction », plus large que la notion d'influence interlinguale, ou «cross-linguistic influence»), l'étude porte aussi sur les coûts de l'alternance ( switch costs ») et sur le contrôle des systèmes plurilingues.

2 Étant donnée la dynamique du traitement de trois langues qui restent simultanément actives, les interactions interlinguales sont inévitables et souvent imprévisibles. Suivant Herdina et Jessner (2002: 29), nous adoptons ici le terme d'interactions interlinguales (" cross-linguistic interaction»), qui comporte une variété d'interactions, y compris le transfert, les interférences, les emprunts, les alternances codiques, etc. En abandonnant la définition assez répandue mais imprécise des interférences comme synonyme du transfert négatif, Herdina et Jessner (2002: 29) distinguent entre le transfert, qui est le phénomène relativement stable de transférer des éléments d'une langue à l'autre, et qui peut être positif ou négatif, et les interférences, qui constituent des interactions dynamiques, non réductibles à une seule langue. Par conséquent, les sources du transfert sont souvent possibles d'identifier, surtout dans le contexte de L2, car en L3 il peut y avoir du transfert de L1 ou de L2, ou bien de L1 et de L2 en même temps (Heine, 2004 ; Näf \& Pfander, 2001). En revanche, les sources des interférences sont souvent impossibles d'identifier, car les interférences sont "le résultat d'une interaction dynamique entre deux ou plusieurs systèmes linguistiques» (Herdina \& Jessner, 2002: 29, notre traduction). Cependant, les interactions interlinguales ne doivent pas forcément être observables. Ringbom (1987: 51) distingue entre les influences interlinguales manifestes et cachées ( overt and covert cross-linguistic influence). Ces premières, divisées en transfert et en 
emprunts, sont basées sur la similarité perçue entre L1 et L2. Par contre, les influences interlinguales couvertes, liées aussi au phénomène de l'évitement, ont lieu là où l'apprenant n'aperçoit pas de similarité entre L1 et L2 et il se base sur les procédures de L1 (Ringbom, 2001 : 51). Par conséquent, il y a des interactions interlinguales qui ne sont pas directement observables mais dont la présence peut être déduite à un certain degré du comportement des sujets, tel que l'évitement, les hésitations, ou bien leurs propres commentaires.

3 Ici, dans l'analyse de la production langagière des participants, nous allons nous concentrer surtout sur les influences ou bien les interactions interlinguales manifestes, car elles peuvent être observées. Or, nous admettons aussi la possibilité de l'apparition d'influences interlinguales cachées et de l'évitement, et ils sont pris en considération là où il y en a des preuves, par exemple, les participants en admettent dans les questionnaires.

4 Les questions de recherche sont les suivantes : premièrement, quels types d'interactions interlinguales ont été observés et dans quelles directions s'opéraient-elles? Deuxièmement, quelles conclusions peut-on en tirer par rapport à l'organisation et au contrôle des répertoires plurilingues?

5 L'étude a été effectuée en contexte universitaire, avec des étudiants suffisamment compétents en L2 et en L3 pour participer à un dialogue relativement spontané dans les deux langues. La seule participante qui ne soit pas étudiante est la participante 3, qui est enseignante-chercheuse, mais ses compétences en L2 et en L3 correspondent à la méthodologie de notre étude. Le contexte de l'étude et les participants sont présentés de façon plus détaillée dans la Section 4 ( cf. infra).

6 À la différence d'autres études sur les coûts de l'alternance (par exemple, Meuter \& Allport, 1999 ; Gollan \& Ferreira, 2009 ; Gullifer, Kroll \& Dussias, 2013), nous n'avons pas utilisé de mots ou bien de phrases séparées, mais des échanges alternés en L2 et en L3. De plus, les stimuli n'étaient pas «préfabriqués», mais l'interaction entre l'enquêteur et les participants incluait la production spontanée de réponses aux questions de l'enquêteur.

7 Certes, la spontanéité était limitée, car c'était l'enquêteur qui imposait généralement les alternances, par exemple, en posant une question en anglais après un échange en allemand. Toutefois, les participants pouvaient formuler les réponses eux-mêmes, ce qui leur permettait d'éviter certaines erreurs d'interférence (par exemple, ne pas prononcer les mots dont ils n'étaient pas sûrs) et, quand ils avaient des difficultés visibles, l'enquêteur pouvait changer de langue ou leur souffler le mot cible si l'alternance, par exemple, à l'anglais, servait à solliciter l'équivalent allemand.

\section{L'alternance codique chez les bi- et plurilingues}

\section{L'activation, le contrôle et la compétition entre les langues}

8 En général, au sein des systèmes bi- et plurilingues, les langues ne sont pas séparées, mais elles constituent les sous-systèmes d'un système plus large de compétence linguistique (Paradis, 1993 : 282). Les interactions entre elles sont donc inévitables. Certes, chaque sous-système peut être sélectivement inhibé (Paradis, 1993 : 282), mais comme l'indiquent les emprunts involontaires à une langue non cible, c'est-à-dire, à une langue autre que la 
langue de l'échange (Poulisse \& Bongaerts, 1994 ; Williams \& Hammarberg, 1998), cette inhibition n'est pas toujours complète.

9 En fait, la disponibilité d'une langue pour l'usage dépend considérablement de son niveau d'activation. Selon Green (1986), une lange peut être sélectionnée (être la langue utilisée dans l'échange), active (qui n'est pas sélectionnée, mais qui a aussi un haut niveau d'activation et qui peut, par exemple, interférer avec la langue sélectionnée) ou dormante (désactivée, non utilisée depuis un certain temps, et qui ne participe donc pas au traitement des langues).

10 Toutefois, à part l'activation de chaque langue dans son entier, il peut y avoir des différences entre l'activation des différents éléments de la langue. Par exemple, le niveau d'activation d'une langue peut permettre sa compréhension, mais pas sa production, car le seuil d'activation nécessaire pour la production est trop haut en raison du manque d'utilisation. En effet, si une unité lexicale n'est pas utilisée pendant longtemps, son seuil d'activation s'élève tellement qu'elle cesse d'être disponible pour la production, mais elle peut rester disponible pour la compréhension, déclenchée par les stimuli externes (Paradis, 1993 : 282-283). En outre, même dans l'utilisation d'une langue sélectionnée, l'autre langue (ou bien les autres langues) n'est désactivée que partiellement et même si « le seuil d'activation est suffisamment élevé pour empêcher les interférences dans la production» (Paradis, 1993 : 284, notre traduction), cela n'empêche pas complètement les emprunts, le mélange des langues ou la compréhension de l'autre langue (Paradis, 1993 : 284).

11 Cela est aussi lié aux habitudes langagières des bi- et plurilingues, car l'usage séparé ou joint des langues dépend souvent des habitudes de la communauté. Comme l'explique Paradis (1993 : 284), les individus qui utilisent régulièrement l'alternance des langues ont le seuil d'activation de la langue non sélectionnée plus bas que ceux qui parlent chaque langue à un groupe monolingue et qui n'ont pas l'opportunité de faire des alternances.

12 Étant donné la désactivation incomplète de la langue non sélectionnée, l'accès au lexique mental n'est pas sélectif, mais les langues rivalisent pour la sélection. Cette compétition est surtout visible dans la compréhension, comme par exemple, la reconnaissance des mots écrits et parlés. Dans la reconnaissance visuelle, la recherche dans le lexique mental n'est " pas compatible avec l'hypothèse de la recherche pré-sélective » (Grainger \& Beauvillain, 1987 : 314, notre traduction), selon laquelle la recherche s'opère dans le lexique d'une langue, puis dans celui de l'autre. De même, le traçage des mouvements oculaires a révélé la coactivation des mots phonologiquement semblables, par exemple, speaker (hautparleur) en anglais et «spichki» (allumettes) en russe (Marian \& Spivey, 2003). Comme le stipule Green (1993 : 260), l'input active simultanément toutes les unités lexicales qui sont compatibles avec celui-ci. Si la structure de certains mots compétiteurs de L1 se chevauche avec celle des mots cibles de L2, la compréhension est inhibée.

La notion de la compétition entre les langues est aussi soutenue par Kroll, Bobb, Misra et Guo (2008), qui ont analysé les résultats d'un certain nombre d'études sur la sélection des langues dans la production bilingue. Ils concluent (Kroll et al., 2008: 426, notre traduction) que les résultats suggèrent que «non seulement les deux langues du bilingue sont activées en parallèle, mais qu'elles rivalisent pour la sélection dans la production orale."

D'une part, le Modèle de la Compétition (The Competition Model), proposé par Bates et McWhinney (1989), n'admet aucun niveau de contrôle stratégique (Green, 1993 : 261); il admet que le savoir est implicite, lorsque le contrôle stratégique exigerait du savoir explicite. Par conséquent, il n'explique pas comment les locuteurs arrivent à exprimer les 
idées pour lesquelles ils manquent de mots en L2, bien qu'ils le fassent effectivement, par exemple, en faisant des paraphrases. Green (1993: 263-264) propose donc le modèle du contrôle inhibitoire (" the inhibitory control model »), où les mots sont marqués (" tagged ») de la langue à laquelle ils appartiennent. Au début, le processus de la sélection augmente l'activation des mots marqués de la langue cible, puis il inhibe les mots marqués de la langue non cible.

Cependant, l'intention de parler une langue ne suffit pas pour inhiber l'activation de l'autre langue (Kroll et al., 2008 : 426). Un rôle dans la sélection d'une langue particulière est joué par un signal de langue («language cue»), par exemple, les informations disponibles dans le contexte linguistique, les buts de la tâche, etc. Toutefois, les bilingues sont capables de résoudre la compétition entre les langues, ce qui ne pourrait pas être expliqué par une approche "déficitaire » du bilinguisme, mais par un modèle qui admet la « sélection par compétition » (« a selection-by-competition model »).

En effet, comme l'ont montré Cedden et Sağın şimşek (2014), le contrôle du système linguistique est encore plus fort chez les trilingues que chez les bilingues, grâce à l'expérience linguistique plus étendue de ces premiers. Plus précisément, l'incorporation de la troisième langue au répertoire linguistique entraîne un coût accru de la ségrégation et de la régulation des langues, mais en définitive elle contribue à l'amélioration du système de contrôle exécutif (Cedden \& Sağın şimşek, 2014 : 565). Une autre expérience qui contribue au développement du contrôle est l'alternance des langues, qui est plus fréquente chez les trilingues que chez les bilingues (Cedden \& Sağın şimşek, 2014 : 565).

En outre, comme l'ont montré Meuter et Allport (1999), les coûts de l'alternance codique sont asymétriques, ou plus précisément, le retour à la langue dominante (L1) après avoir utilisé L2 exige plus d'effort que le passage de L1 à L2. Tout paradoxal que cela paraisse, le passage à L2 exige l'inhibition active de la langue dominante L1, qui, à son tour, requiert davantage d'activation quand on la sélectionne de nouveau.

Or, il est possible que les coûts de l'alternance dépendent du type de stimuli. Meuter et Allport (1999) ont utilisé des mots séparés: les participants devaient nommer les numéraux en L1 et en L2. En revanche, Gullifer, Kroll et Dussias (2013) ont utilisé des phrases entières dans un ordre mixte (anglais, anglais, espagnol, espagnol, etc.), où les participants devaient nommer les cognats utilisés dans les phrases. Gullifer et al. (2013) sont arrivés à la conclusion que l'accès lexical bilingue est non sélectif, malgré l'intention de limiter la production à une seule langue. Dans leur étude, il n'y a pas eu de coût mesurable du passage d'une langue à l'autre (Gullifer et al., 2013 : 9). Pourtant, sur la base d'autres études, Gullifer et al. (2013: 11) admettent qu'il y a effectivement des coûts d'alternance, surtout dans le cas des alternances imprévisibles (Monsell et al. 2003, dans Gullifer et al. 2013: 11), mais aussi dans les alternances prévisibles (Meuter \& Allport, 1999; Monsell et al. 2003, dans Gullifer et al. 2013: 11). Toutefois, la prévisibilité des alternances joue un rôle important dans la récupération mesurée en termes du temps de réaction après une alternance des tâches ("task switching») : la récupération est plus rapide - et donc le coût de l'alternance est moins élevé - quand les alternances initiées par le chercheur sont prévisibles, mais elle est affaiblie quand les alternances s'opèrent de façon aléatoire (Monsell et al., 2003, dans Gullifer et al., 2013 : 11). Autrement dit, quand les alternances imposées par la méthodologie de l'étude sont prévisibles pour les participants, le coût cognitif de l'alternance est moindre que dans le cas des alternances imprévisibles, qui forcent les participants à réagir immédiatement. Dans la présente étude et dans les études citées ici, il s'agit de tâches linguistiques, comme par exemple, les 
échanges oraux (la présente étude), ou bien la mémorisation de phrases suivie ou non par des jugements métalinguistiques (Guzzardo Tamargo, 2012, dans Gullifer et al., 2013 : 11). Or, précisément dans la présente étude, étant données les différences entre L2 et L3 au niveau de la compétence linguistique des participants et de leur expérience langagière, nous admettons que l'usage de L2 et celui de L3 peuvent être considérés comme des tâches distinctes.

Toutefois, malgré les coûts cognitifs, les bilingues n'évitent pas les alternances, mais par contre, ils alternent volontiers les langues (Gollan \& Ferreira, 2009: 659). Dans les alternances volontaires, ils ont tendance à utiliser une langue plutôt que l'autre. Or, dans l'étude de Gollan et Ferreira (2009), la production était limitée aux mots séparés, car les stimuli étaient des images que les participants devaient nommer en L1 ou en L2.

En effet, tout en admettant qu'à la différence de leur étude, dans les contextes naturels une séquence d'alternances artificiellement régulière est improbable, Gullifer et al. (2013 : 11) supposent que si les bilingues contrôlent l'ordre des alternances eux-mêmes, les coûts de l'alternance sont encore plus bas. En revanche, dans les études utilisant d'autres tâches, les coûts de l'alternance étaient considérables et, en plus, ils variaient d'une tâche à l'autre. Comme le concluent Gullifer et al. (2013: 11), cela suggère que les coûts de l'alternance deviennent visibles quand les ressources de mémoire sont considérablement épuisées. En revanche, le coût de l'alternance est bas quand le contexte linguistique, notamment celui de la phrase, est suffisant pour surmonter l'inhibition de la langue antérieurement désactivée.

21 En outre, dans le cas des alternances volontaires un rôle important est joué, d'une part, par la disponibilité des mots et, d'autre part, par les facteurs pragmatiques, bien qu'à la différence de l'interaction naturelle, les raisons pragmatiques de l'alternance, comme par exemple la personnalisation, soient limitées dans la tâche expérimentale (Gollan \& Ferreira, 2009: 647-648). La fréquence des alternances est plus haute chez les bilingues plus équilibrés, ce qui indique que l'alternance devient plus facile avec l'augmentation de la compétence linguistique (Gollan \& Ferreira, 2009: 647 et 662). Nous pouvons donc admettre que les coûts de l'alternance dépendent de plusieurs facteurs, comme par exemple, la prévisibilité des alternances et l'expérience linguistique des participants ; ils peuvent dont varier d'une étude à l'autre, voire d'un participant à l'autre.

\section{La notion de mode langagier}

Comme les langues jouent souvent des rôles particuliers dans les répertoires linguistiques des locuteurs et des communautés bi- et plurilingues, le choix d'une langue ou de l'autre ne se fait normalement pas au hasard, mais selon certaines règles, comme le statut de chaque langue, sa fonction dans la communauté, etc. (Grosjean, 1982: 135-145). Bien entendu, l'alternance codique et le mélange des langues suivent certaines règles aussi.

Afin d'expliquer les différentes façons de mélanger les langues ou bien de les utiliser séparément, Grosjean a proposé la notion de mode langagier («language mode »), qui est défini par "l'état de l'activation des langues du bilingue et des mécanismes de traitement des langues à un moment donné dans le temps » (Grosjean, $2001: 3$, notre traduction). En mode monolingue, la langue non cible n'est activée que faiblement et le bilingue parle seulement la langue cible, par exemple, quand l'interlocuteur est monolingue. Par contre, en mode bilingue les deux langues sont actives, quoique la langue de base (A) soit plus active que l'autre langue (B), qui n'est pas la langue principale de traitement. Le mode 
bilingue a lieu quand les interlocuteurs sont bilingues et qu'ils comprennent les alternances et les emprunts à la langue $\mathrm{B}$. Finalement, il y a un mode intermédiaire, où la langue B est partiellement activée, par exemple, quand l'interlocuteur ne la connaît pas bien, ou quand il n'aime pas mélanger les langues (Grosjean, 2001 : 3-4).

Comme le souligne Grosjean (2001 : 13), étant donné l'importance du mode langagier dans le comportement des bilingues, cette variable devrait être contrôlée dans les études sur le bilinguisme, notamment quand ce n'est pas la variable principale. Par exemple, les chercheurs en bilinguisme prétendent souvent avoir observé de nombreux cas d'interactions interlinguales et d'éléments de la langue non cible. Or, selon Grosjean (2001: 13-14), nous ne serons jamais capables de distinguer parfaitement entre les interférences, les alternances codiques et les emprunts dans la parole bilingue. Ainsi, ce qui pourrait sembler être une interférence peut en effet être une «structure invitée» (« guest structure », Grosjean, $2001: 14)$ produite par le locuteur qui sait que l'interlocuteur comprend le langage mixte, contenant des éléments de L1 et de L2. Comme les bilingues savent que, par exemple, le chercheur connait aussi l'autre langue, ils sont souvent en mode intermédiaire ou bien bilingue pendant les enregistrements. En revanche, une personne trilingue peut être en mode monolingue, bilingue ou trilingue (Grosjean, 2001 : 17).

La notion de mode langagier a été questionnée par Dijkstra et Van Hell (2001). Selon eux, le mode langagier serait extrêmement sensible aux facteurs contextuels implicites, par exemple, la seule présence d'une étudiante en français activerait le français des participants. Or, comme ils ont utilisé des mots isolés comme stimuli, il est possible que les conditions de leur étude aient été différentes de la communication naturelle entre les bilingues, qui utilisent les mots et les phrases en contexte.

Pourtant, des preuves en faveur de l'existence des modes langagiers ont été fournies par l'étude de Dunn et Fox Tree (2014), où il y avait des différences entre le rejet des pseudomots en mode monolingue et en mode bilingue. Or, le mode de langue est particulièrement visible quand la compétence linguistique est contrôlée. Effectivement, la dominance d'une langue par rapport à l'autre joue un rôle important et, par exemple, un bilingue chez qui l'espagnol est la langue fortement dominante peut ne jamais être capable d'inhiber son espagnol complètement et rester dans un mode monolingue anglais, ce qui serait possible chez un bilingue plus équilibré (Dunn \& Fox Tree, 2014: 612).

27 Le mode langagier constitue donc un facteur important, mais lié aussi à d'autres facteurs, tels que la compétence dans chaque langue. Il est possible, par conséquent, que malgré le niveau de contrôle qui élimine les interférences visibles (cf. Cedden \& Sağın şimşek, 2014, supra), un trilingue ne puisse jamais être en mode monolingue L3, car L1 et peut-être aussi L2 peuvent résister à l'inhibition et rester actives.

\section{Les modèles de la production bi- et plurilingue}

Sans doute, étant donné que le traitement des langues au sein des répertoires bi- et plurilingues est plus complexe que dans les répertoires monolingues, les modèles de la production de la parole par les bi- et plurilingues doivent prendre en considération de nombreux facteurs, comme par exemple, la compétence linguistique des locuteurs et l'activation des langues. Selon le modèle proposé par de Bot (1992), le plan de la parole (« 
speech plan ») est préparé dans la langue sélectionnée (cf. Section 2.1. supra) et, en parallèle, dans la langue active (de Bot, 1992 : 13). Or, dans la plupart des cas, seulement la langue sélectionnée participe à la production.

Pendant la production de la parole, les mots seront choisis initialement dans la langue sélectionnée, ou bien dans la langue active si c'est nécessaire, ou en dernier ressort dans la langue dormante, avec une perte considérable de temps comme résultat. (De Bot, $1992: 13$, notre traduction).

Selon De Bot (1992: 13), ce genre de traitement rend possible l'alternance fréquente et effectuée avec aisance.

Au niveau du conceptualisateur (« conceptualizer»), c'est-à-dire, le module qui prépare le message préverbal, il y a deux étapes de planification de la parole : la macroplanification, qui est indépendante de la langue, et la microplanification, qui est déjà spécifique à la langue, parce que les concepts diffèrent d'une langue à l'autre (par exemple, «here »/ " there» en anglais et "aquí»/ "ahí»/ "allí» en espagnol, selon la distance entre le locuteur et l'objet). Le module suivant, dit le formulateur (« formulator »), est spécifique à la langue (de Bot, 1992: 21). L'alternance codique, selon de Bot (1992: 19, notre traduction), exige " un sous-système qui contienne des éléments lexicaux des différentes langues "; les mots « déclic » («triggerwords», De Bot, 1992:19) sont similaires en forme aux mots de l'autre langue et, par conséquent, ils provoquent les alternances.

Or, le modèle proposé par de Bot est critiqué par Poulisse et Bongaerts (1994: 40), qui stipulent que la préparation de deux plans d'énoncés exigerait trop d'énergie et de ressources cognitives de la part du locuteur. Selon eux, l'information concernant le choix de la langue devrait être ajoutée au message préverbal. Suivant Green (1993), ils admettent que les lemmes sont marqués de la langue à laquelle ils appartiennent et ils stipulent que l'information sur le choix de la langue est ajoutée au message préverbal, ce qui contribue à l'activation des unités lexicales particulières (Poulisse \& Bongaerts, 1994 : 41).

En revanche, sur la base de l'alternance codique dans la parole plurilingue, Williams et Hammarberg (1998) ont proposé un modèle qui attribue différents rôles aux langues faisant partie du répertoire plurilingue. Ils distinguent quatre types d'alternances : EDIT (l'autocorrection, le début d'un tour, etc.), META (les commentaires métalinguistiques, les demandes d'aide, etc.), INSERT (l'utilisation des mots des langues autres que L3 pour solliciter l'équivalent en L3), et WIPP ("Without Identified Pragmatic Purpose», ou les alternances qui ne servent aucun but pragmatique identifiable, Williams \& Hammarberg, 1998: 295). Sur la base des langues participant à ces différents types d'alternances, Williams et Hammarberg $(1998: 304)$ attribuent aux langues de la participante trois rôles : le fournisseur par défaut («default supplier»), qui est un fournisseur interlingual et qui fournit des emprunts, le fournisseur intralingual (par exemple, quand l'apprenant essaie de créer des mots de L3 sur la base d'autres mots de la même langue) et le rôle instrumental, qui sert à faire des commentaires métalinguistiques, demander de l'aide, etc.

Les résultats de leur étude montrent que ces fonctions dépendent de la langue; par exemple, l'anglais (L1) jouait le rôle INSTRUMENTAL et il servait à exprimer les commentaires métalinguistiques, alors que l'allemand (L2), qui a fourni le plus d'alternances Wipp, était le FOURNISSEUR PAR DÉFAUT, dû à son niveau d'activation, au niveau de compétence de la participante, à la similarité avec L3 (suédois) et à son statut de langue étrangère. 

etc.). Le transfert fréquent de mots outils de L1 (finnois) et de L2 (suédois) en L3 (anglais) a aussi été observé par Ringbom (1987). Comme le conclut Ringbom (1987: 128), les locuteurs font moins attention aux mots outils, en se concentrant sur les mots lexicaux. De Angelis (2005: 12) attribue l'utilisation de L2 en tant que fournisseur par défaut à la perception de la correction ("perception of correctness») et à l'association du caractère étranger («association of foreignness »). En effet, les emprunts à la langue maternelle sont souvent perçus comme incorrects, les apprenants ont donc tendance à transférer des mots et des structures d'une langue étrangère à l'autre. Certes, la langue maternelle fait partie de la psychotypologie (terme de Kellerman, 1987) des apprenants et elle leur sert de source de transfert, mais dû à leur statut de langues étrangères, deux langues étrangères peuvent être perçues comme plus proches l'une de l'autre que de la langue maternelle. En outre, l'association du caractère étranger et la perception de la correction peuvent être plus fortes que le marquage («tagging») des mots et, par conséquent, un mot marqué comme, par exemple, «italien», peut pour autant être regardé par l'apprenant comme espagnol, comme s'il était marqué comme « espagnol » (De Angelis, $2005: 13)$.

nt, il faut aussi prendre en considération les facteurs affectifs. Comme l'a montré Gabryś-Barker (2006: 112), dans les protocoles verbaux, les passages à la langue maternelle ont lieu dans le cas des réactions émotionnelles.

Nous pouvons donc admettre que dans les répertoires linguistiques des apprenants de L3, aussi des participants à notre étude, les différentes langues jouent des rôles différents et que cela se reflète dans la production de L2 et de L3.

\section{L'étude}

\section{Participants}

37 L'étude a été effectuée avec dix-sept participants au moins trilingues (seize étudiants et une enseignante universitaire), dont quatorze étaient de sexe féminin et trois de sexe masculin.

Ils possédaient les combinaisons de langues suivantes: polonais-allemand-anglais (8 participants), polonais-anglais-allemand (6), polonais-français-anglais (1), polonaisanglais-russe (1) et allemand-polonais-anglais (1). Cependant, ce dernier a révélé sa combinaison de langues dans le questionnaire après l'enregistrement, auquel il avait participé comme si l'allemand était sa L2 et l'anglais sa L3. En outre, il habite et étudie en Pologne et parle polonais quotidiennement. Nous pouvons donc admettre qu'il est le plus probablement membre de la minorité allemande en Pologne et que son niveau de compétence peut effectivement être plus haut en polonais qu'en allemand.

Les langues étaient classées sur la base de la compétence linguistique des participants : L2 était leur langue étrangère la mieux maîtrisée, alors que L3 était moins bien maitrisée, mais les niveaux de compétence dans les deux langues permettaient aux sujets de participer à des interactions orales.

L'étude a été effectuée en contexte universitaire, à l'Université Polonia à CzĘstochowa (participants 1 à 7) et à l'Université d'Opole (participants 8 à 17). Bien que la population échantillon soit assez variée, ceci est dû à la disponibilité limitée des étudiants trilingues

Recherches en didactique des langues et des cultures, 13-2 | 2016 
suffisamment compétents en L2 et en L3 et à la diversité des répertoires plurilingues en général. En fait, comme l'ont remarqué Van Gelderen et al. (2003: 23), les groupes d'apprenants de L3 sont souvent plus hétérogènes que ne l'exige la méthodologie de l'étude. Or, malgré la diversité des participants et de leurs répertoires, nous admettons que l'étude peut fournir des observations intéressantes sur les interactions interlinguales et sur la gestion des répertoires plurilingues. En fait, parmi les étudiants avec la combinaison polonais-allemand-anglais, à part ceux qui étudiaient l'allemand et l'anglais en parallèle, il y a eu trois étudiantes (participantes 1, 2 et 5) qui faisaient leurs études en maîtrise d'anglais après avoir obtenu des diplômes de maîtrise d'allemand. De même, l'enseignante-chercheuse (participante 3), tout en étant docteur en linguistique anglaise, avait d'abord étudié la philologie russe, mais dû à l'utilisation quotidienne de l'anglais et à l'attrition du russe, elle a indiqué sa combinaison de langues comme polonais-anglaisrusse.

En général, d'une part, le groupe n'était pas suffisamment homogène pour faire des calculs statistiques. D'autre part, cette diversité de répertoires langagiers a fourni des observations qui n'auraient peut-être pas été possibles dans un groupe homogène, étudiant la même L2 et la même L3 dès le début.

\section{Méthode}

La méthode utilisée dans l'étude comportait des interactions orales en L2 et en L3 entre les participants et l'enquêteur. Les participants tiraient au sort une question relativement simple en L2, par exemple, leur sport préféré, leur animal préféré, s'ils aimaient voyager, etc. ${ }^{1}$ On commençait par des échanges en L2, en admettant que ce serait plus facile pour les participants. Or, après quelques échanges, l'enquêteur passait à L3, ce qui exigeait l'inhibition de L2, le retour à L2 pouvait donc être plus difficile. En outre, les échanges n'étaient pas toujours L2-L3-L2-L3, mais leur ordre était imprévisible pour les participants, comme par exemple, L2-L2-L2-L3-L3-L2-L3-L3 (participante 1), L2-L2-L2-L2L3-L3-L2-L2-L2-L3-L3-L3-L3-L2-L2 (participante 5; ici, les échanges étaient plus nombreux, mais aussi plus courts, car trois d'entre eux ont servi à solliciter les mots cibles et la participante a aussi demandé s'il lui fallait continuer).

43 À la différence de l'étude de Cedden \& Sağın şimşek (2014), qui ont utilisé des listes de questions courtes, composées à l'avance, dans notre étude seule la première question, qui était tirée au sort, était préparée. Certes, dans l'étude de Cedden \& Sağın şimşek, l'ordre des langues sur les listes était plutôt aléatoire (par exemple, turc, allemand, turc, anglais, allemand, turc, etc.), mais dès que le participant avait répondu à une question, les auteures passaient à la question suivante. En revanche, dans notre étude les questions consécutives en L2 et en L3 se référaient à ce que les participants venaient de dire, si bien que la langue et le contenu étaient pratiquement imprévisibles, au moins pour les participants. Nous pouvons donc supposer que cela augmentait encore les coûts de l'alternance.

44 Les dialogues ont été enregistrés, puis transcrits selon les normes de Blanche-Benveniste \& al. (1990), légèrement adaptées (voir Annexe 2 à la fin de l'article). En totalité, les dixsept enregistrements durent deux heures et quinze minutes, mais la longueur des enregistrements particuliers varie en fonction du participant, da sa volonté de parler L2 et $\mathrm{L} 3$, du contenu de ses réponses, etc. 
Après les dialogues, les participants ont rempli un questionnaire concernant leurs biographies langagières, leurs stratégies d'apprentissage de L2 et de L3, y compris l'utilisation du transfert, et les difficultés rencontrées au cours de l'étude.

\section{Les résultats}

En général, les résultats indiquent que la coactivation de trois langues exige un haut niveau de contrôle et que, même chez les trilingues, qui sont habitués à contrôler leurs répertoires linguistiques, les interactions interlinguales sont inévitables. Effectivement, nous avons observé des interactions intra- et interlinguales chez tous les participants, y compris chez l'enquêteur.

Les interactions interlinguales sous forme d'emprunts et d'alternances codiques étaient relativement peu nombreuses, généralement deux ou trois par personne. Dans le cas de deux participants, nous n'avons pas observé d'interactions visibles de ce type. Ici, la catégorie d'emprunts et d'alternances codiques comporte les éléments d'une langue autre que la langue cible, par exemple, des mots de L1 ou de L2 en L3. Elle comporte donc les mots utilisés par hasard, les emprunts stratégiques, ainsi que les alternances qui servent à demander un mot à l'enquêteur. En revanche, elle ne comporte pas les erreurs de transfert ou d'interférence qui ne contiennent pas d'éléments d'une langue non cible, mais, par exemple, des traductions littérales d'expressions de structures grammaticales d'une autre langue. Les nombres d'emprunts et d'alternances codiques observés chez les participants sont présentés dans le Tableau 1.

Tableau 1 - Nombres d'interactions observées sous forme d'alternances et d'emprunts

\begin{tabular}{|l|l|l|l|l|l|l|l|l|l|l|l|l|l|l|l|l|l|}
\hline Participant & 1 & 2 & 3 & 4 & 5 & 6 & 7 & 8 & 9 & 10 & 11 & 12 & 13 & 14 & 15 & 16 & 17 \\
\hline Alternances/emprunts & 5 & 5 & 2 & 3 & 2 & 1 & 2 & 0 & 1 & 1 & 2 & 2 & 3 & 3 & 0 & 1 & 1 \\
\hline
\end{tabular}

Moyenne $: 34: 17=2$

Comme le montre le Tableau 1, les alternances et les emprunts étaient peu nombreux, de zéro à cinq cas par personne, où la moyenne était de deux alternances ou bien emprunts par personne.

Étant donné l'activation de toutes les langues, les participants étaient en mode trilingue, mais selon les consignes, ils essayaient d'inhiber les langues non cibles pour parler chaque fois la langue sélectionnée par l'enquêteur. Par conséquent, ils utilisaient les langues non sélectionnées pour demander certains mots à l'enquêteur (Meta, la souscatégorie Elicit, dans la terminologie de Williams \& Hammarberg, 1998), quoique nous ayons aussi observé des alternances Wipp, qui étaient des mots outils ou bien des mots lexicaux.

Pourtant, il faut souligner que certaines interférences n'étaient pas visibles dans la production, car les participants les ont inhibées. En revanche, elles se manifestaient par des pauses et la recherche du mot cible. Certes, une pause ne doit pas forcément refléter une interférence inhibée, car elle peut également être due, par exemple, à la recherche d'un mot dans le lexique mental. Or, si les participants le confirment eux-mêmes, certaines hésitations sont effectivement attribuables à l'inhibition d'interférences. Par 
exemple, la participante 5 (polonais-allemand-anglais) a dit "some things ", l'enquêteur lui a soufflé le mot « ingredients » qu'elle cherchait, puis dans le questionnaire elle a admis que le mot «Zutaten» lui était venu à l'esprit. De même, la participante 3 a écrit qu'elle avait voulu dire «between » au lieu de « МЕЖДУ» et qu'en général, elle avait eu du mal à récupérer les mots russes, car elle utilisait l'anglais au travail tous les jours. Il est aussi possible que les participants aient eu d'autres interférences de ce type, mais ils ne les ont pas révélées et nous avons seulement observé des hésitations.

Pour cette raison, en général, le nombre des interférences ne peut être qu'approximatif. De même, certaines erreurs sont difficiles à classer, car elles peuvent être également des faux départs ou des reformulations qui ne peuvent être attribués aux interférences. Parmi les alternances observées, nous pouvons distinguer plusieurs types. Dans les exemples, les directions des interactions interlinguales sont indiquées par des flèches. Premièrement, les alternances Wipp, c'est-à-dire, "Without Identified Pragmatic Purpose» (Hammarberg \& Williams, 1998: 295), ou sans but pragmatique identifié, étaient relativement fréquentes et elles suggèrent que l'activation de certains mots était plus forte que les mécanismes de contrôle et le marquage («tagging ») des mots.

Exemple 1.1:

(Wipp, participante 5, polonais-allemand-anglais ; L2 $\rightarrow \mathrm{L}$ 3)

« the art how we eat »

(l'art dont on mange ; cible : la façon dont on mange ; erreur non corrigée)

Le mot allemand «art» (façon, genre) a été utilisé en anglais, sans que l'étudiante s'en aperçoive; ceci est un exemple de ce que De Angelis (2005: 14) appelle "le changement de système» ("system shift »), c'est-à-dire, la perception d'un mot d'une langue étrangère non cible comme appartenant à la langue cible.

Exemple 1.2:

(Wipp, participante 1, polonais-allemand-anglais ; L2 $\rightarrow$ L3)

« when I + in the future + I am: + ready mit + with my English study »

(quand je + dans l'avenir + je suis : + prête avec [c'est-à-dire, quand je termine - note de Teresa Maria Wlosowicz] + avec mes études en anglais)

Ici, la préposition «mit» (avec) constitue une alternance Wipp que l'étudiante corrige immédiatement. Or, toute la construction est une traduction littérale de l'allemand, « wenn ich mit meinem Englischstudium fertig bin »; par contre, en anglais on dirait : « when I complete (or : finish) my English studies».

Exemple 1.3:

(Wipp, participante 1, polonais-allemand-anglais ; L3 $\rightarrow$ L2)

«ich war + im Urlaub + im + äh + in France + und + auch + in Frankreich » (rires)

(j'ai été + en vacances + en + euh + en France (anglais) + et + aussi + en France)

L'alternance Wipp est un mot lexical de L3 inséré en L2. L'étudiante l'a 
corrigé dès qu'elle s'est rendu compte d'avoir utilisé un mot de la langue étrangère non cible.

Exemple 1.4 :

$$
\begin{aligned}
& \text { (Wipp, participante 17, polonais-allemand-anglais ; L2 } \rightarrow \text { L3) } \\
& \text { « he + didn't said for example + er + Armee + aber + er + but + ar- + army " } \\
& \text { (il + ne disait pas par exemple + euh + armee + mais + euh + mais + ar- + army) }
\end{aligned}
$$

En parlant anglais (L3), l'étudiante a inséré un mot allemand ( «aber »), qu'elle a corrigé à «but». Or, le mot "aber» est, en effet, incorrect, car la juxtaposition de deux alternatives dont la première est niée en allemand exige la conjonction "sondern». «Aber» a probablement été une interférence du polonais ou de l'anglais, qui, à la différence de l'allemand, ne font pas la différence entre les deux types de «mais». Du point de vue du modèle de De Bot (1992), le message préverbal était déjà préparé pour l'anglais, ne prenant pas en considération l'existence de deux concepts correspondant à « mais » en allemand. Par conséquent, L2, en tant que fournisseur par défaut, a fourni l'équivalent allemand "de base», et pas l'équivalent qui aurait été plus correct si la phrase avait été formulée en allemand. Cela indique que, bien qu'un mot allemand se soit glissé dans l'énoncé en anglais, aucun plan alternatif en allemand n'a été préparé. En outre, l'étudiante a prononcé « army » /' $\alpha$ : ml/ comme /'ærmı/; il est possible que cette prononciation soit due à la représentation assez répandue parmi les apprenants polonais qu'en anglais la lettre «a » est toujours prononcée comme /æ/. Deuxièmement, nous avons observé des alternances qui servaient à faire des commentaires métalinguistiques (Meta Non Elicit), mais celles-ci étaient effectivement rares.

Exemple 2.1

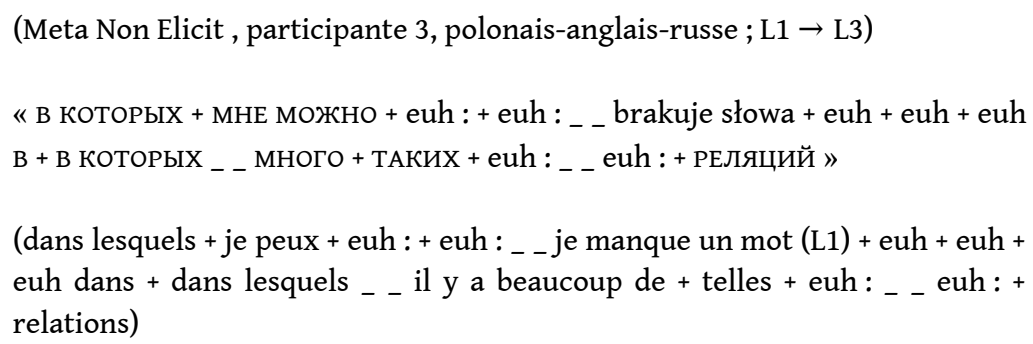

La participante informe l'enquêteur qu'il lui manque un mot, mais elle continue à le chercher. Or, le mot «РЕЛЯцИА (ryelatsa) n'existe pas en russe; la participante l'a inventé sur le base des mots anglais «relation » et «relationship», ou bien "relacja » en polonais, quoique le mot cible russe soit « отношениЕ» (atnashenye) оu « Связь» (svyaz'). Troisièmement, les alternances qui servaient à demander le mot cible à l'enquêteur (Meta Elicit) étaient, en revanche, plus fréquentes. Nous pouvons supposer que, comme les participants savaient que l'enquêteur parlait leur L1, L2 et L3, ils n'inhibaient pas complètement les langues non cibles et ils restaient donc en mode trilingue, en admettant que les alternances seraient aussi comprises et qu'ils obtiendraient de l'aide.

Exemple 3.1 (participante 4, polonais-français-anglais ; L2 $\rightarrow$ L3) :

" if I remember + er: + by the + dinde »

(si je me rappelle bien + euh : + par la + dinde) 


\section{anglais.}

Exemple 3.2 (participante 4, polonais-français-anglais ; L2 $\rightarrow \mathrm{L} 3$ ) :

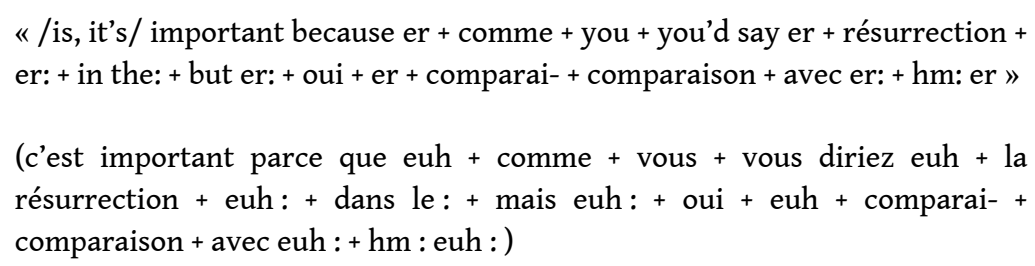

57 L'étudiante cherchait l'expression « in comparison to » et l'enquêteur la lui a donnée. Or, ce tour contient aussi deux alternances Wipp, «comme » et "oui », qui soutiennent le constat de Ringbom (1987) que, comme les locuteurs se concentrent sur les mots lexicaux, ils peuvent faire moins attention aux mots outils. Par conséquent, si un mot français était fortement activé, il pouvait détourner les mécanismes de contrôle, qui se concentraient sur la vérification des mots lexicaux.

Exemple 3.3 (participante 5, polonais-allemand-anglais ; L3 $\rightarrow$ L2) :

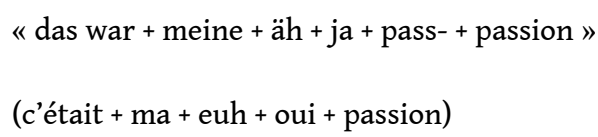

En cherchant le mot «Leidenschaft» (passion) en allemand (L2), l'étudiante n'a pu se rappeler que le mot « passion» en anglais (L3).

Exemple 3.4 (participante 7, polonais-anglais-allemand ; L2 $\rightarrow \mathrm{L} 3$ )

" auf Englisch pumpkin + auf Deutsch + leider wei- + weiß ich nicht »

(en anglais pumpkin + en allemand + malheureusement je ne sais + je ne sais pas)

L'étudiante ne connaissait pas le mot «Kürbis» («citrouille » en allemand), elle a donc utilisé l'équivalent anglais, en espérant que l'enquêteur comprenne son intention communicative et qu'elle lui souffle le mot cible.

Exemple 3.5 (participant 16, allemand-polonais-anglais ; L2 $\rightarrow$ L3, ou bien L1 $\rightarrow$ L3; le statut du polonais dans son répertoire linguistique a été analysé dans la Section 4.1.)

« they are + hm + not erm + jak to jest zgrani »

(ils sont $+\mathrm{hm}+$ pas erm + comment dire ils ne savent pas bien coopérer)

60 L'étudiant est passé au polonais pour demander à l'enquêteur une expression liée à l'équipe de football. En fait, « zgrani » est un mot polonais qui est difficile à traduire et qui exige souvent une paraphrase. Le fait qu'il l'a dit en polonais suggère qu'en allemand il n'y a probablement pas non plus d'équivalent.

Quatrièmement, un certain nombre d'emprunts reflète le transfert stratégique, qui, dans ce cas, est négatif, car il se manifeste par des erreurs. En revanche, le transfert positif n'est pas directement observable, car ses résultats sont des mots et des expressions correctes. Nous ne pouvons donc pas être sûre si une expression correcte, disons, en L3, est une traduction littérale de L1 ou de L2, ou bien si le participant la connaît en L3. Dans 
ce cas, la seule possibilité d'identifier le transfert positif est l'analyse des commentaires des participants.

Exemple 4.1 (participante 13, polonais-allemand-anglais ; L2 $\rightarrow$ L3)

《 maybe + Krakau + with + Wawel + Castle » (peut-être + Cracovie + avec + le château + de Wawel)

Il est possible que la participante n'ait pas eu accès au mot anglais Cracow (Cracovie), c'est pourquoi elle a utilisé le nom allemand. Or, plus tard dans le dialogue, elle a dit «Cracow ", alors « Krakau » a peut-être été aussi une alternance Wipp.

Exemple 4.2 (participante 5, polonais-allemand-anglais ; L3 $\rightarrow$ L2)

« das wilde Leben »

(les animaux sauvages, littéralement « la vie sauvage »)

Ceci est probablement une traduction littérale du mot anglais « wildlife »; en allemand, on dirait plutôt «Wildtiere » (les animaux sauvages).

Exemple 4.3 (participante 7, polonais-anglais-allemand ; L3 $\rightarrow$ L2 $\rightarrow$ L3)

Participante : « ich habe das Wort (rires) + hm (j’ai le mot + hm)

Enquêteur : « vielleicht auf Englisch » (peut-être en anglais)

Participante : « er + recept $($ euh + recept)»

En fait, une recette de cuisine s'appelle "recipe» en anglais; l'étudiante a créé un mélange des mots «recipe» en anglais et «Rezept» en allemand. Autrement dit, la récupération du mot cible allemand a été bloquée par un mot qui semblait être anglais, mais qui était en effet un mélange interlingual germano-anglais. De surcroît, nous avons observé des interférences au niveau syntaxique, qui indiquent que si le plan de l'énoncé est préparé dans une langue, notamment en L2, les mots de L3 remplissent les « lacunes » prévues pour les mots de L2 et pas celles qu'ils rempliraient si le plan était préparé en L3.

Exemple 5.1 (participante 10, polonais-allemand-anglais ; L2 $\rightarrow$ L3)

"you can all the time hear the national languages » (on peut entendre les langues nationales tout le temps)

En anglais, on dirait plutôt "you can hear the national languages all the time ", alors que " you can all the time hear » est une interférence possible de l'allemand, où l'ordre des mots avec le verbe à la fin serait utilisé : « man kann die nationalen Sprachen die ganze Zeit hören ».

Exemple 5.2 (participante 2, polonais-allemand-anglais ; L2 $\rightarrow \mathrm{L} 3$ )

« in German better are the Abiturienten »

(en allemand les élèves en classe du bac sont meilleurs)

L'ordre des mots a été influencé par l'ordre des mots allemand, où le verbe arrive en deuxième position et, si l'adverbe le précède, le sujet doit le suivre. En anglais, on dirait plutôt: "The A-level students are better at German». Par ailleurs, après le mot "Abiturienten ", l'étudiante a commencé à parler allemand; il est donc possible que la préparation de l'énoncé avec l'ordre des mots allemand et le mot «Abiturienten » ait 
soudain augmenté l'activation $\mathrm{du}$ sous-système allemand dans son répertoire linguistique.

Exemple 5.3 (participante 7, polonais-anglais-allemand ; L1 $\rightarrow$ L2)

« because they + would be + curious + er + what is it »

(parce qu'ils + seraient + curieux + euh + qu'est-ce que c'est)

65 À la différence de l'anglais, où il n'y a pas d'inversion dans les questions indirectes et où la structure correcte serait "they would be curious about what it is", en polonais l'ordre des mots dans les questions directes et indirectes est le même. Nous pouvons supposer qu'en se concentrant sur le contenu, la participante a utilisé la procédure de L1 au lieu d'appliquer la règle syntaxique de L2.

Finalement, bien que rares, il y a eu des alternances de type affectif :

Exemple 6.1 (participante 12, polonais- anglais-allemand ; L1 $\rightarrow$ L3)

«weil + äh + äh + ich + ah : + Boże »

(parce que + euh + euh + je + euh $:+$ mon Dieu $)$

67 En s'efforçant de formuler une phrase en allemand, l'étudiante a exprimé son énervement en polonais; cela confirme le constat de Gabryś-Barker (2006) que les expressions affectives sont utilisées souvent dans la langue maternelle. Pourtant, une autre étudiante a dit: "oh God ich verwechsle diese zwei Sprachen " (mon Dieu, je confonds ces deux langues). Apparemment, l'anglais, qui est non seulement la langue étudiée par la participante, mais aussi la langue mondiale, présente dans de nombreux films, chansons, etc., fournit au polonais des interjections qui sont souvent utilisées par les jeunes et qui semblent être moins fortes que les interjections polonaises.

68 À part les interférences comportant des mots entiers, nous avons observé des interférences aux niveaux inférieurs à celui du mot, notamment les interférences phonologiques (par exemple, 'important' en anglais, prononcé comme /' $\varepsilon^{\sim}$ portant/) et morphologiques, soit intralinguales (*schmeckhaft, au lieu de "schmackhaft", *Medieval Ages, au lieu de "Middle Ages», etc.), soit interlinguales, par exemple, "tressieren », un mélange de «tresować » en polonais et «dressieren » en allemand.

En ce qui concerne les coûts de l'alternance, ils sont reflétés, d'une part, par les hésitations dans la production orale des participants après les alternances et, d'autre part, par les commentaires faits par les participants eux-mêmes. En fait, huit participants sur dix-sept ont constaté dans le questionnaire que le passage d'une langue à l'autre leur avait posé des difficultés particulières. Ils ont écrit, entre autre, qu'ils avaient encore pensé dans la langue non cible, qu'il leur avait été difficile de récupérer le vocabulaire allemand après avoir parlé anglais (ou l'inverse). Une participante a même eu du mal à se rendre compte du fait que l'enquêteur lui avait déjà parlé dans une autre langue. Une neuvième participante a aussi constaté que l'allemand avait toujours été présent au fond de sa tête, même quand elle avait essayé de penser en anglais. Cela indique que, bien que les trilingues sachent contrôler leurs systèmes linguistiques, le passage d'une langue à l'autre exige un coût cognitif considérable. 


\section{Conclusion}

70 En général, le nombre relativement faible d'alternances et les nombreuses pauses et hésitations indiquent que les participants cherchaient à contrôler les interférences et à parler la langue cible choisie par l'enquêteur. Ainsi, bien qu'ils aient été en mode bi- ou trilingue, ils essayaient de limiter la production à la langue cible imposée par l'enquêteur, malgré la coactivation de L2, de L3 et de L1, et de passer à une autre langue seulement quand c'était nécessaire, c'est-à-dire, soit au moment de passer à la nouvelle langue cible, soit pour demander un mot à l'enquêteur. Autrement dit, ils exerçaient un contrôle conscient sur leurs répertoires plurilingues pour parler la langue cible et pour éviter les emprunts et les alternances non désirées. Cela confirme la conclusion de Ceddem \& Sağın şimşek (2014) que, grâce à leur expérience linguistique considérable, les trilingues savent contrôler leurs répertoires linguistiques et limiter le nombre d'interférences. Or, comme l'indiquent les alternances Wipp, cela n'était pas toujours possible, car l'activation de certains mots était plus forte que les mécanismes de contrôle.

Comme le montrent les exemples, les interactions interlinguales se sont opérées dans plusieurs directions, c'est-à-dire, de L1 à L2, de L1 à L3, de L2 et à L3 et de L3 à L2. Nous n'avons pas observé d'influences de L2 ou de L3 sur L1, mais c'est à cause du fait que les dialogues aient été effectués en L2 et en L3, mais pas en L1. Le fournisseur par défaut était généralement L2, mais nous avons aussi observé un certain nombre d'interférences de L3 en L2 (comme par exemple, "das wilde Leben", « recept»), ce qui indique que chez la plupart des participants, la relation entre L2 et L3 est assez équilibrée. Les exceptions sont les participantes 3 et 4 , qui n'utilisent pas régulièrement le russe et l'anglais respectivement, et la participante 5, qui enseigne l'allemand et qui a écrit qu'il était toujours présent au fond de sa tête, même quand elle pensait en anglais. De même, le passage automatique à l'allemand déclenché par un seul emprunt à l'allemand suggère que chez la participante 2, qui enseigne aussi cette langue, l'allemand L2 est effectivement la langue étrangère dominante et fortement activée. En revanche, chez la participante 1, nous avons observé des interactions dans les deux directions, de L2 à L3 et à l'inverse, ce qui suggère que, tout en étant enseignante d'allemand, elle a une relation plus équilibrée entre son anglais et son allemand.

Nous pouvons supposer que, si L2 avait été leur langue étrangère fortement dominante, ils auraient fait beaucoup plus d'erreurs d'interférence de L2 en L3, et très peu voire aucune interférence de L3 en L2. De même, les alternances involontaires auraient été probablement uniquement des mots de L2 utilisés en L3. Certes, comme dans l'étude de Williams \& Hammarberg (1998), L2 semble être le fournisseur par défaut de tous les participants, sauf pour les deux participantes qui n'ont pas fait d'alternances autres que celles imposées par le chercheur. Or, bien que la compétence des participants en L2 soit plus haute qu'en L3 (sinon il serait impossible de les classer comme L2 et L3, mais plutôt comme Lx et Ly, cf. Cenoz, 2000: 40), ils contrôlent leurs répertoires linguistiques relativement bien et il est possible que l'exercice du contrôle contribue à l'augmentation de l'équilibre. Nos résultats soutiennent le constat de Herdina \& Jessner (2002 : 29), selon lequel les interférences sont bidirectionnelles et non réductibles à une seule langue et elles caractérisent les systèmes plus équilibrés, alors que le transfert, où les structures sont transférées d'une langue (ex.:L1) vers l'autre (ex.:L2), est caractéristique des systèmes non équilibrés. Certes, étant donné l'hétérogénéité des systèmes plurilingues, 
un plurilingue ne peut pas être équilibré au même degré qu'un bilingue équilibré, mais nous pouvons supposer qu'il peut y avoir des relations plus ou moins équilibrées entre les sous-systèmes du répertoire linguistique, par exemple, entre L1 et L2, entre L2 et L3, etc.

En ce qui concerne l'organisation et le contrôle des systèmes plurilingues, les résultats de cette étude indiquent qu'en effet, les langues sont représentées comme sous-systèmes d'un système plus large et que les possibilités de contrôler l'interférence sont limitées. En fait, comme toutes les trois langues des participants étaient activées, nous avons observé des interférences dans pratiquement toutes les directions (sauf de L2 ou L3 en L1, mais l'étude se concentrait sur la production de L2 et de L3) et de plusieurs types. Comme Ringbom (1987), nous avons observé de nombreuses alternances Wipp qui comportaient des mots outils (" aber », «comme», «ja», « mit », etc.), ce qui suggère que les mots outils sont plus difficiles à contrôler, mais dans le même temps, les alternances Wipp avec les mots lexicaux indiquent que chaque mot qui arrive au niveau d'activation nécessaire à la production peut être produit, indépendamment de la langue cible, qui ne doit pas être un signal (cue) suffisant (cf. Kroll et al. 2008). Autrement dit, l'activation d'un mot de la langue non cible peut dépasser la quantité d'énergie disponible au locuteur pour l'inhibition de celui-ci. Il faut souligner que la production de dialogues entiers peut fournir davantage d'informations sur l'organisation et le traitement des langues que les études qui utilisent des mots isolés, et il serait souhaitable de continuer les recherches dans ce domaine, en améliorant de plus en plus la méthodologie. Malheureusement, dû aux limites de longueur de l'article, nous ne pouvons pas présenter ici les dialogues entiers, ce qui serait possible dans l'annexe à un livre. Nous espérons pour autant que les exemples inclus dans le présent article permettent d'illustrer plusieurs cas intéressants d'interactions interlinguales au sein des répertoires plurilingues, et que les recherches futures à plus grande échelle, avec des populations échantillons plus grandes, fourniront des données suffisamment nombreuses pour effectuer des calculs statistiques et obtenir des résultats quantitatifs significatifs.

Finalement, bien que la présente étude soit psycholinguistique plutôt que didactique, il est possible d'en tirer aussi quelques conclusions applicables à la didactique des langues, notamment du plurilinguisme. En particulier, l'étude confirme l'inséparabilité et l'interaction entre les langues des plurilingues. Cela remet en question l'approche monolingue du bi- et plurilinguisme, selon laquelle chaque langue peut être séparément évaluée, comme si le trilingue était la somme de trois monolingues (Grosjean, 1985, De Angelis \& Selinker, 2001), alors qu'il est un locuteur avec une combinaison de langues unique, déterminée par son expérience linguistique. Certes, en préparant les étudiants, par exemple, aux examens, il faut les rendre conscients des règles de chaque langue, pour qu'ils apprennent à contrôler leurs répertoires linguistiques, mais d'un autre côté, l'exigence de la correction ne devrait pas pénaliser les apprenants plurilingues. En même temps, afin de pouvoir bien évaluer la production linguistique des apprenants, il serait souhaitable que les enseignants soient eux-mêmes plurilingues. D'une part, ils pourraient mieux identifier les interférences et aider les apprenants à les éliminer autant que possible. D'autre part, les enseignants de L3 comprendraient mieux les difficultés rencontrées par les apprenants et ils pourraient mieux les aider à maîtriser deux ou plusieurs langues étrangères. 


\section{BIBLIOGRAPHIE}

Bates, E. \& MacWhinney, B. (1989). «Functionalism and the Competition Model ». In

MacWhinney, B. \& Bates, E. (dir.). The Crosslinguistic Study of Sentence Processing. Cambridge :

Cambridge University Press.

Blanche Benveniste, C., Bilger, M., Rouget, C. \& van den Eynde, K. (1990). Le français parlé : études grammaticales. Paris : Centre National de la Recherche Scientifique.

Cedden, G.\& Sağın şimşek, Ç. (2014). « The impact of a third language on executive control processes ». International Journal of Bilingualism, vol. 18, $\mathrm{n}^{\circ} 6$, pp. 558-569.

Cenoz, J. (2000). « Research on Multilingual Acquisition ». In Cenoz, J. \& Jessner, U. (dir.). English in Europe : The Acquisition of a Third Language. Clevedon/Buffalo/Toronto/Sydney : Multilingual Matters.

De Angelis, G. (2005). « Multilingualism and non-native lexical transfer : An identification problem ». International Journal of Multilingualism, vol. 2, n¹, pp. 1-25.

De Angelis, G. \& Selinker, L. (2001). « Interlanguage Transfer and Competing Linguistic Systems in the Multilingual Mind ». In Cenoz, J., Hufeisen, B. \& Jessner, U. (dir.). Cross-Linguistic Influence in Third Language Acquisition: Psycholinguistic Perspectives. Clevedon : Multilingual Matters, Ltd.

De Bot, K. (1992). Applied Linguistics, vol. 13, n 1, pp. 1-24.

Dijkstra, T., van Hell, J. (2001). « Testing the Language Mode hypothesis using trilinguals ». Communication présentée à la deuxième Conférence Internationale sur l'Acquisition de la Troisième Langue et le Trilinguisme, Fryske Akademy, 13-15 September 2001 (CD-ROM).

Dunn, A.L. \& Fox Tree, J.E. (2014). « More on language mode ». International Journal of Bilingualism, vol. $18, \mathrm{n}^{\circ} 6, \mathrm{pp} .605-613$.

Gabryś-Barker, D. (2006). « Language Activation in the Thinking Processes of a Multilingual Language User ». International Journal of Multilingualism, vol. 3, n², pp. 105-124.

Gollan, T.H. \& Ferreira, V.S. « Should I Stay or Should I Switch? A Cost-Benefit Analysis of Voluntary Language Switching in Young and Aging Bilinguals ». Journal of Experimental Psychology: Learning, Memory and Cognition, vol. 35, n³, pp. 640-665.

Grainger, J. \& Beauvillain, C. (1987). « Language blocking and lexical access in bilinguals ». Quarterly Journal of Experimental Psychology: Human Experimental Psychology, vol. 39A, pp. 295-319.

Green, D.W. (1986). « Control, Activation and Resource». Brain and Language, vol. 27, pp. 210-223.

Green, D.W. (1993). « Towards a Model of L2 Comprehension and Production ». In Schreuder, R. \& Weltens, B. (dir.). The Bilingual Lexicon. Amsterdam/Philadelphia : John Benjamins Publishing Company.

Grosjean, F. (1982). Life with Two Languages. An Introduction to Bilingualism. Harvard University Press : Cambridge, Massachusetts and London, England.

Grosjean, F. (1985). « The bilingual as a competent but specific speaker-hearer ». Journal of Multilingual and Multicultural Development, vol. 6, pp. 467-477. 
Grosjean, F. (2001). « The bilingual's language modes. » In Nicol, J. (dir.). One Mind, Two Languages: Bilingual Language Processing. Oxford : Blackwell.

Gullifer, J.W., Kroll, J.F. \& Dussias, P.E. (2013). « When language switching has no apparent cost : lexical access in sentence context. » Frontiers in Psychology, vol. 24, Article 278, pp. 1-13. Disponible en ligne. www.frontiersin.org

Guzzardo Tamargo, R.E. (2012). Linking Comprehension Costs to Production Patterns During the Processing of Mixed Language. University Park : The Pennsylvania State University.

Heine, L. (2004). « Mögliches und Unmögliches : Zur Erforschung von Transfererscheinungen ». In Hufeisen, B. \& Marx, N. (dir.). Beim Schwedischlernen sind Englisch und Deutsch ganz hilfsvoll. Untersuchungen zum multiplen Sprachenlernen. Frankfurt a.M. : Peter Lang Verlag.

Herdina, P. \& Jessner, U. (2002). A Dynamic Model of Multilingualism. Perspectives of Change in Psycholinguistics. Clevedon : Multilingual Matters Ltd.

Kellerman, E. (1987). Aspects of Transferability in Second Language Acquisition. Nijmegen : Katholieke Universiteit Nijmegen.

Kroll, J.F., Bobb, S.C., Misra, M. \& Guo, T. (2008). « Language selection in bilingual speech : Evidence for inhibitory processes ». Acta Psychologica, vol. 128, pp. 416-430.

Marian, V. \& Spivey, M. (2003). « Bilingual and monolingual processing of competing lexical items ». Applied Psycholinguistics, vol. 24, pp. 173-193.

Meuter, R.F.I. \& Allport, A. (1999). « Bilingual Language Switching in Naming: Assymetrical Costs of Language Selection ». Journal of Memory and Language, vol. 40, pp. 25-40.

Näf, A., Pfander, D. (2001). « <Springing of > a <bruck> with an elastic <sail> - Deutsches im Englischen von französischsprachigen Schülern ». Zeitschrift für Angewandte Linguistik, vol. 35, pp. 5-37.

Paradis, M. (1993). « Multilingualism and Aphasia ». In Blanken, G., Dittman, J., Grimm, H., Marshall, J.C. \& Wallesch, C.W. (dir.). Linguistic Disorders and Pathologies. An International Handbook. Berlin/ New York : Walter de Gruyter.

Poulisse, N. \& Bongaerts, T. (1994). « First Language Use in Second Language Production ». Applied Linguistics, vol. $15, \mathrm{n}^{\circ} 1$, pp. 36-57.

Ringbom, H. (1987). The Role of the First Language in Foreign Language Learning. Clevedon : Multilingual Matters.

Van Gelderen, A., Schoonen, R., de Glopper, K., Hulstijn, J., Snellings, P., Simis, A., Stevenson, M. (2003). « Roles of linguistic knowledge, metacognitive knowledge and processing speed in L3, L2 and $\mathrm{L} 1$ reading comprehension. A structural equation modeling approach ». International Journal of Bilingualism, vol. 7, n 1 , pp. 7-25.

Williams, S. \& Hammarberg, B. (1998). «Language Switches in L3 Production: Implications for a Polyglot Speaking Model ». Applied Linguistics, vol. 19, n³, pp. 295 - 333.

\section{ANNEXES}

\section{Annexe 1 Questions utilisées dans l'étude}

1. Quel est votre hobby ? Pourquoi? 
2. Quel est votre animal préféré et pourquoi?

3. Préférez-vous Noël ou Pâques ? Pourquoi ?

4. Quel est votre livre préféré et pourquoi?

5. Aimez-vous voyager? Pourquoi ou pourquoi pas?

6. Que feriez-vous si vous gagniez un million de dollars?

7. Quel est votre sport préféré et pourquoi?

8. Quel est votre film préféré et pourquoi ?

\section{Annexe 2 Normes de transcription des enregistrements}

+ pause courte

- - pause moyenne

- - - pause longue

/ / / / interruption assez longue

$\mathrm{X}$ syllabe incompréhensible

XXX séquence de syllabes incompréhensibles

/le, la/ deux transcriptions possibles, la première jugée la plus probable

/le, ø/ hésitation entre une écoute et rien

pass- amorce d'un mot

\section{NOTES}

1. La liste des questions utilisées dans l'étude est présentée dans l'Annexe 1 à la fin de l'article. Comme les questions étaient les mêmes dans toutes les L2s, l'Annexe 1 présente uniquement les questions en français.

\section{RÉSUMÉS}

Le but de la présente étude est une investigation des alternances codiques entre L2 et L3 dans les interactions orales dans plusieurs combinaisons de langues en contexte universitaire. Bien que les interactions interlinguales (le transfert, les interférences, les emprunts, etc., Herdina \& Jessner, 2002) se soient opérées dans plusieurs directions, elles étaient relativement peu nombreuses, ce qui reflète le niveau de contrôle des répertoires linguistiques par les participants. Les résultats indiquent que chez la plupart des participants la relation entre L2 et L3 est relativement équilibrée, ce qui peut être dû à leur expérience dans l'exercice du contrôle sur le répertoire plurilingue. Toutefois, comme l'admettent les participants eux-mêmes, le passage 
d'une langue à l'autre leur a posé des difficultés considérables, ce qui reflète le coût cognitif des alternances codiques, notamment celles imposées par la méthodologie de l'étude.

The purpose of the present study is an investigation of code-switching between L2 and L3 in oral interaction in several language combinations in a university context. Even though crosslinguistic interaction (transfer, interference, borrowings, etc., Herdina and Jessner, 2002) was observed in several directions, there were relatively few of them, which reflects the participants' level of control over their language repertoires. The results indicate that in most of the participants the relationship between L2 and L3 is relatively balanced, which may be due to their experience in exercising control over their language repertoires. However, as the participants themselves admit, switching from one language to the other caused them considerable difficulty, which reflects the cognitive cost of code-switching, especially that imposed by the research methodology.

INDEX

Mots-clés : plurilinguisme, alternance codique, interférences, contrôle des répertoires plurilingues

Keywords : plurilingualism, code-switching, interference, control of plurilingual repertoires

\section{AUTEUR}

\section{TERESA MARIA WLOSOWICZ}

Université d'Ostrava, Ostrava, République Tchèque

Institut de Philologie Anglaise et Américaine, Faculté de Philosophie, Realní 5, 70103 Ostrava, République Tchèque.

Teresa Maria Wlosowicz est docteur en Sciences du Langage et enseignante-chercheuse à l'Université d'Ostrava en République Tchèque. Elle a obtenu le grade de docteur en co-tutelle à l'Université de Silésie à Katowice, Pologne, et à l'Université de Strasbourg en 2009. Ses recherches portent sur le plurilinguisme, le pluriculturalisme, la psycholinguistique, l'acquisition et la didactique des langues, l'anglais comme langue internationale et la traduction. Elle enseigne aussi l'anglais des affaires.

Courriel : melomane.plurilingue[at]gmail.com 